Physicians and Surgeons, New York, New York, USA; ${ }^{2}$ Gastroenterology, North Shore University, New York, USA; ${ }^{3}$ Internal Medicine, North Shore University, New York, USA; ${ }^{4}$ Internal Medicine, NYMC Richmond, New York, USA

Introduction Hepatitis $\mathrm{E}$ is essentially an oral fecal infection with high prevalence in developing countries. There is limited data available on its prevalence in Urban US. The study evaluates the prevalence of hepatitis E antibody IgG and IgM in New York in patients with chronic liver disease.

Methods $440(n=440)$ were divided into two groups: group $A$; control 140 patients without any stigmata of liver disease. Group B, 300 patients with history of liver disease including hepatitis B 125/ 300 (41\%), Chronic hepatitis C 60/300 (20\%), fatty liver 70/300 (23\%), Alcoholic liver disease 29/300 (10\%), HBV HCV co infection $9 / 300$ (3\%), auto immune hepatitis $2 / 300$ (0.6\%), PBC /PSC 5/300 $(2 \%)$. HEV antibody IGM and IGG were measured with HEV genotyping. 22/300 (7\%) patients were liver transplant recipients, 6/ $300(2 \%)$ patients were kidney transplant recipients. 43/300 (14\%) patients were Intra venous Drug Abuser. 20/300 (7\%) received Blood products.

Results History and genotypic characteristics

\begin{tabular}{lllll}
\hline Characteristics & $\begin{array}{l}\text { Chronic } \\
\text { hepatitis C }\end{array}$ & Chronic hepatitis B & Fatty liver & $\begin{array}{l}\text { Alcoholic liver } \\
\text { disease }\end{array}$ \\
\hline IVDU & $36 / 43(83 \%)$ & $7 / 43(16 \%)$ & NA & NA \\
Promiscuity & $23 / 42(52 \%)$ & $10 / 42(23 \%)$ & NA & $9 / 42(2 ! \%)$ \\
Transfusion & $6 / 20(30 \%)$ & $14 / 20(70 \%)$ & NA & NA \\
Genotype & & & & \\
G1 & $2 / 30(7 \%)$ & $60 / 69(87 \%)$ & $3 / 10(30 \%)$ & $1 / 13(7 \%)$ \\
G2 & $1 / 30(3 \%)$ & $8 / 69(11 \%)$ & $3 / 10(30 \%)$ & $4 / 13(30 \%)$ \\
G3 & $26 / 30(87 \%)$ & $1 / 69(1 \%)$ & $4 / 10(40 \%)$ & $8 / 13(62 \%)$ \\
G4 & $1 / 30(3 \%)$ & NA & NA & NA \\
G4 & NA & NA & NA & NA \\
\hline
\end{tabular}

Conclusion Results: Group (A) HEV IgG positive 13\% (18/140), Group B HEV IgG positive 40.6\% (122/300) including 3.7\% (4/122) having both IgM and IgG positive. The prevalence of HEV IgG was $54 \%(12 / 22)$ in the liver transplant recipient group and 33\% (2/6\%) kidney transplant recipient group. The study demonstrates the prevalence of hepatitis E infection in New York and HEV antibody in CLD. Including transplant donors and recipients. Question remains the impact and progression of acute or chronic liver disease with concomitant HEV in pre, peri, and post liver transplant recipient. Larger study needs to validate.

Competing interests None declared

\section{PM0-171 A PILOT OF TARGETED CASE FINDING FOR HEPATITIS B VIRUS (HBV) AND HEPATITIS C (HCV) IN THE BRITISH SOUTH ASIAN COMMUNITY}

doi:10.1136/gutjnl-2012-302514b.171

\footnotetext{
${ }^{1,2} \mathrm{~S}$ Mcpherson, ${ }^{*}{ }^{3} \mathrm{~S}$ Moses, ${ }^{3} \mathrm{M}$ Valappil, ${ }^{4} \mathrm{~S}$ Rehman, ${ }^{1} \mathrm{C}$ Miller, ${ }^{1} \mathrm{~K}$ Baxter, ${ }^{5} \mathrm{~A}$ Saeed, ${ }^{6} \mathrm{~K}$ Shafiq, ${ }^{1,2} \mathrm{M}$ Hudson, ${ }^{1,2} \mathrm{M}$ Bassendine. 'Liver Unit, Freeman Hospital, Newcastle upon Tyne, UK; ${ }^{2}$ Institute of Cellular Medicine, Newcastle University, Newcastle upon Tyne, UK; ${ }^{3} H P A$, Newcastle upon Tyne, UK; ${ }^{4}$ Broadway Medical Centre, Newcastle upon Tyne, UK; ${ }^{5}$ Queen Elizabeth Hospital, Gateshead, UK; ${ }^{6}$ Sunderland Royal Hospital, Sunderland, UK
}

Introduction Chronic HBV (cHBV) and HCV (cHCV) are frequent causes of cirrhosis and liver cancer. $\mathrm{cHBV}$ and $\mathrm{cHCV}$ are prevalent in subjects from South Asian countries and many infected individuals are unaware of their condition. A previous case finding pilot for $\mathrm{HBV}$ in the British-Chinese community of the NE of England found that $9 \%$ of subjects tested were $\mathrm{HBsAg}$ positive. ${ }^{1}$ Targeted screening for
HBV and HCV is recommended by the AASLD ${ }^{2}$ and the European Liver Patients Association ${ }^{3}$ in subjects born in endemic areas (HBsAg prevalence $\geq 2 \%$ ). However, there are no current UK guidelines. Our aim was to apply AASLD recommendations to British South Asian community of NE of England.

Methods Members of the NE South Asian community were invited to attend screening sessions at a Newcastle Mosque and the Sunderland Bangladeshi Community Centre. Dry blood spots were obtained by finger prick and tested for $\mathrm{HBsAg}, \mathrm{HBcAb}$ (Abbott ARCHITECT ${ }^{\circledR}$ ) and HCV antibody (Roche + confirmed by Biorad). $\mathrm{HBsAg}$ and/or HCV Ab positive individuals were advised to undergo confirmatory testing and be referred for specialist assessment.

Results To date, 329 subjects were screened in four sessions (median age 39 , range $6-83 ; 75 \%$ male). The screened subjects were most frequently born in Bangladesh (49\%) followed by Pakistan $(36 \%)$, the UK $(14 \%)$ or other countries $(6 \%) .1 .8 \%$ of subjects were $\mathrm{HBsAg}$ positive indicating $\mathrm{cHBV} .2 .8 \%$ of subjects had past infection with $\mathrm{HBV}$ ( $\mathrm{HBs} \mathrm{Ag}$ negative, $\mathrm{HBcAb}$ positive). $1 \%$ of subjects were $\mathrm{HCV}$ $\mathrm{Ab}$ positive (all born in Pakistan). The prevalence of $\mathrm{cHBV}$ was 3\% for individuals born in Pakistan and $1.5 \%$ for those born in Bangladesh. 3\% of individuals born in Pakistan were HCV Ab positive. Only $4 \%$ of subjects reported previous vaccination against HBV.

Conclusion Undiagnosed cHBV is prevalent in the South Asian community of NE England, although the prevalence was lower than in the British-Chinese community. These results provide evidence for a targeted case finding programme for HBV and HCV in subjects born in South Asia.

Funding This study was supported by a Gilead UK and Ireland Fellowship and Livernorth.

Competing interests None declared.

\section{REFERENCES}

1. McPherson S, et al. Gut 2011;60(Supp 2):A25

2. Lok A, McMahon B. Hepatology 2009;50:1-36.

3. Piorkowsky N. J Hepatol 2009;51:1068-73.

\section{PM0-172 DIRECT NON-INVASIVE SERUM MARKERS OF LIVER FIBROSIS PREDICT FIBROSIS EVOLUTION IN CHRONIC HEPATITIS C BUT ARE INCREASED BY INTERFERON-BASED THERAPY}

doi:10.1136/gutjnl-2012-302514b.172

${ }^{1} \mathrm{~S}$ Tanwar, ${ }^{*}{ }^{1} \mathrm{P} M$ Trembling, ${ }^{2} \mathrm{E}$ Ellis, ${ }^{2} \mathrm{~J}$ Parkes, ${ }^{3} \mathrm{C}$ Herold, ${ }^{4} \mathrm{D}$ Schuppan, ${ }^{1}$ W M C Rosenberg. ${ }^{1}$ Centre for Hepatology, University College London (UCL), London, UK; ${ }^{2}$ Public Health Sciences and Medical Statistics, University of Southampton, Southampton, UK; ${ }^{3}$ University of Erlangen, Erlangen, Germany; ${ }^{4}$ Department of Internal Medicine, University of Mainz, Mainz, Germany

Introduction Liver biopsy remains the reference standard for the detection of liver fibrosis. While non-invasive markers of liver fibrosis have been validated in chronic hepatitis $\mathrm{C}$, their performance during interferon-based therapy is not established.

Methods 70 previous non-responders to interferon based therapy for chronic hepatitis C (40 male, age range $24-67$, mean 48.7) were recruited from five centres. Patients were randomised to receive pegylated interferon with or without silymarin for 24 months as an exploratory antifibrotic therapy. All patients underwent a liver biopsy and ELF tests (HA, P3NP, TIMP-1) prior to, and after treatment (month 0 and 24). Changes in histological fibrosis stages before and after therapy ( 0 to 24 months) were compared with changes in marker scores before, during and after therapy (0 to 24 months).

Results Mean ELF score prior to therapy was 9.32 (SD 1.10), during therapy 9.91 (SD1.18) and after therapy 9.53 (SD 1.27). ELF scores 
were significantly higher during therapy than when compared to both before therapy $(p<0.00001)$ and after therapy $(p=0.0002)$ but levels before therapy were not significantly different from post therapy. Elevated scores on therapy were attributable to an increase in mean HA $(p<0.0002)$ and P3NP $(p<0.01)$ levels on therapy, whereas a significant change in mean TIMP-1 during therapy was not seen. These elevations were seen in all patients regardless of changes in histological fibrosis after therapy ( $n=20$ decrease, $n=25$ no change, $\mathrm{n}=35$ increase in Ishak stage). However, individual changes in TIMP1 $(r=0.239, p=0.04)$ and changes in ELF $(r=0.315$, $\mathrm{p}=0.004$ ) from pre- to post-therapy levels were found to correlate with the change of Ishak fibrosis stage before and during treatment. Conclusion During interferon-based therapy, levels of HA and P3NP and the ELF score rise globally and subsequently fall to values similar to those seen prior to therapy regardless of fibrosis evolution, while TIMP1 levels remained unaffected. However, ELF scores pre and post therapy did accurately reflect changes in histology. This suggests that ELF scores and non-invasive panels incorporating $\mathrm{HA}$ and P3NP should be interpreted with caution during interferonbased therapy.

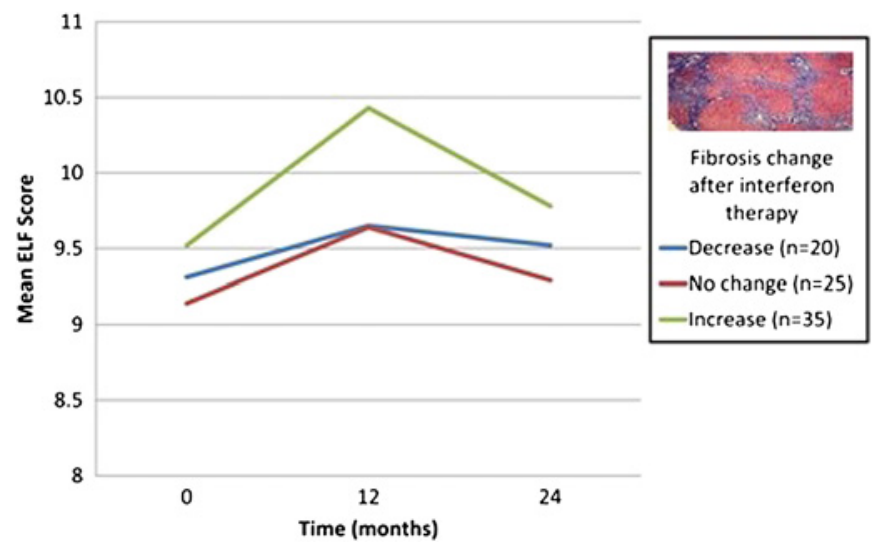

Abstract PM0-172 Figure 1 Fibrosis evolution compared to changes in mean ELF score during interferon therapy.

Competing interests None declared.

\section{PM0-173 DISTRICT GENERAL HOSPITAL NETWORKS CAN PROVIDE SAFE AND EFFECTIVE HEPATITIS C TREATMENT: RESULTS OF A 4-YEAR AUDIT}

doi:10.1136/gutjnl-2012-302514b.173

${ }^{1} \mathrm{~T}$ Hydes, ${ }^{*}{ }^{2} \mathrm{H}$ Allen, ${ }^{2} \mathrm{~S}$ Al-Shamma, ${ }^{3} \mathrm{C}$ Hovell, ${ }^{1} \mathrm{~N}$ Sharer, ${ }^{2} \mathrm{E}$ Williams. ${ }^{1}$ Gastroenterology \& Hepatology, Poole Hospital NHS Foundation Trust, Poole, UK; ${ }^{2}$ Gastroenterology \& Hepatology, Royal Bournemouth and Christchurch Hospitals NHS Foundation Trust, Bournemouth, UK; ${ }^{3}$ Gastroenterology \& Hepatology, Dorset County Hospital NHS Foundation Trust, Dorchester, UK

Introduction Chronic Hepatitis C (CHC) treatment is well described in the context of Randomised Controlled Trials (RCTs). ${ }^{1}$ Whether these findings can be extrapolated to treatment programmes delivered by nurse specialists working in District General Hospitals (DGHs) is unclear.

Methods The Dorset Viral Hepatitis Network has a catchment area of 750000 people. Patients are assessed and treated in three DGHs by a team of nurse specialists working under the supervision of four lead clinicians. Between January 2007 and January 2011 standard of care for $\mathrm{CHC}$ treatment was Ribavirin and Pegylated Interferon $\alpha 2 \mathrm{a}$ given for 24 weeks (G2/3 patients) to 48 weeks (G1/4). A retrospective analysis of the network's reference database was undertaken focusing on treatment naïve patients.
Results In total 207 treatment naïve patients received antiviral therapy. Mean age at time of treatment was 43 years (20-66); 74\% (154) were male and 67\% (139) acquired CHC through injection drug use. G1 patients represented 49\% (102) of the cohort; $3 \%$ (6) were Hepatitis B/HIV co-infected and 95\% (196) were Caucasian. A clinical or histological diagnosis of cirrhosis was present in 8\% (16). In total $12 \%$ (24) moved out of area or were lost to follow-up within 24 weeks of completing treatment. Based on intention to treat, Sustained Virological Response rates (undetectable HCV RNA in serum 24 weeks post treatment) were comparable to those derived from $\mathrm{RCT}^{1}$ data (Abstract PMO-173 table 1 ). Non-response was observed in $11 \%(11 / 102), 5 \%(5 / 98)$ and $14 \%(1 / 7)$ of G1, G2/3 and G4 patients respectively. Breakthrough or relapse was observed in $18 \%(18 / 102), 13 \%(13 / 98)$ and $14 \%(1 / 7)$ of $\mathrm{G} 1, \mathrm{G} 2 / 3$ and G4 patients respectively. Overall $1 \%$ (3) of patients discontinued treatment as a result of a laboratory abnormality and 12\% (24) because of other medical complications or side effect intolerance. These proportions are comparable to those observed in RCTs $(\mathrm{p}=0.735, \mathrm{p}=0.146)$.

Abstract PM0-173 Table 1 Comparison of SVR rates between centres

\begin{tabular}{llll}
\hline Genotype & SVR in DGH practice & SVR in RCT $^{\mathbf{1}}$ & p Value $\dagger$ \\
\hline Genotype 1 & $46 \%(47 / 102)$ & $41 \%(241 / 583)$ & 0.37 \\
Genotype $2 / 3$ & $60 \%(59 / 98)$ & $68 \%(194 / 285)$ & 0.16 \\
Genotype 4 & $57 \%(4 / 7)$ & $58 \%(14 / 24)$ & 0.96
\end{tabular}

*51\% Received Peg IFN $\alpha 2 \mathrm{a}$.

†Derived from $\chi^{2}$ test.

Conclusion Specialist nurses supported by a network of DGHs can deliver a high quality Hepatitis $C$ service across a broad geographical area. These findings are encouraging when considering a move towards community based CHC management.

Competing interests None declared.

\section{REFERENCE}

1. Fried MW, Shiffman ML, Reddy KR, et al. Peginterferon alfa-2a plus ribavirin for chronic hepatitis C virus infection. New Engl J Med 2002; 347:975-82.

\section{PM0-174 INTRAHEPATIC NATURAL KILLER CELL PHENOTYPING AND FUNCTIONAL ANALYSIS BY FINE NEEDLE ASPIRATION IN CHRONIC HCV INFECTION}

doi:10.1136/gutjnl-2012-302514b.174

T Pembroke, ${ }^{*}$ E Wang, A Gallimore, A Godkin. School of Medicine, Cardiff University, Cardiff, UK

Introduction Hepatitis $\mathrm{C}$ virus (HCV) infection results in chronic infection in the majority of subjects, indicating viral immunoevasion strategies. Treatment with interferon alpha (IFN $\alpha$ ) stimulates the immune system but the role of NK cells remains unclear.

Methods Intra-hepatic NK cells were obtained from $20 \mathrm{HCV}$ infected donors prior to treatment and 16 non-viral chronic liver disease (CLD) patients along with paired peripheral blood samples. NK phenotype (CD16, NKp30, NKp46, NKG2D and NKG2A) and functional profile (Ki67, CD107a, IFN- $\gamma$ and Granzyme B) was assessed by flow cytometry. In a separate cohort of $8 \mathrm{HCV}$ patients, who had completed treatment, rate of viral clearance was calculated and pre-treatment peripheral blood NK phenotype and CD107a expression in response to increasing stimulation was measured. At low-level stimulation peripheral blood mononuclear cells (PBMCs) were incubated overnight with $50 \mathrm{u} / \mathrm{ml}$ IFN $\alpha$ and exposed to Huh7. Five target cells and at maximal stimulation PBMCs were incubated with $1000 \mathrm{u} / \mathrm{ml} \mathrm{IFN} \alpha$ and $\mathrm{K} 562$ target cells. 